\title{
Adicción a internet e inteligencia emocional: análisis de una relación
}

Enviado: 28 de septiembre de 2020 / Aceptado: 27 de octubre de 2020

Publicado: 30 de diciembre de 2020

JUAN CARLOS RODRÍGUEZ RODRÍGUEZ

Universidad de Almería, España

jucar61@ual.es

JOSEFINA RODRÍGUEZ-GÓNGORA

Universidad de Almería. España jrg293@inlumine.ual.es

JOSÉ ANTONIO RODRÍGUEZ RODRÍGUEZ

Universidad de Almería. España jrr962@inlumine.ual.es

RESUMEN

La accesibilidad y el aumento del uso de internet ha llevado en los últimos años a realizar numerosas investigaciones, dirigidas principalmente a establecer si el uso de este recurso es o no saludable para los ciudadanos. Se han correlacionados numerosas variables, entre ellas la Inteligencia Emocional y se ha intentado determinar el papel que representa dentro de las posibles adicciones. En este estudio intentamos ver la influencia que tiene la inteligencia emocional sobre el uso abusivo de internet o del móvil Para ello, hemos encuestado a 547 sujetos, que nos han contestado a dos cuestionaros para medir el uso abusivo del móvil e internet y la inteligencia emocional. A pesar de haber obtenido resultados con puntuaciones dentro de parámetros normales, se ha encontrado una correlación negativa entre la inteligencia emocional y el uso abusivo de internet y móvil, con diferenciación significativa entre hombres y mujeres. Se propone la realización de investigacio-

\section{ABSTRACT}

Internet addiction and emotional intelligence: analysis of a relationship

The accessibility and the increase in the use of the internet have been carried out in recent years to carry out new research, mainly aimed at establishing the use of this resource or unhealthy for citizens. The variables that we have been frequently correlated, including Emotional Intelligence and an attempt has been made to determine the role it plays within possible additions. In this study, we try to see the influence that emotional intelligence has on the abusive use of the internet or mobile. For this, we have a sample of 547 subjects who have answered two questions to measure the abusive use of mobile phones and the internet and emotional intelligence. Despite having obtained normal results, a negative correlation has been found between emotional intelligence and Internet and mobile use, with a significant differentiation between 
nes mas profundas, con diferentes instrumentos que corroboren los resultados, y la puesta en marcha de programas preventivos encaminados a mejorar los niveles de inteligencia emocional de los ciudadanos, con el fin de paliar las posibles consecuencias aversivas del uso abusivo de internet.

Palabras Clave: inteligencia emocional, adicción, internet, abuso del móvil, jóvenes. the men's and women's. It is proposed to carry out more in-depth research, with different instruments that corroborate the results and to launch preventive programs aimed at improving the levels of emotional intelligence of citizens-harm, to mitigate the possible aversive consequences of use abusive internet.

Keywords: emotional intelligence, addiction, internet, mobile abuse, youth.

\section{INTRODUCCIÓN}

Hablar de adicción al uso de internet y/o el móvil puede resultar controvertido, por lo que hemos querido utilizar otra terminología mas apropiada, en este caso Uso abusivo. Han sido diversos los autores que han visto la necesidad de estudiar estas conductas y se han realizado diferentes estudios y elaborados cuestionarios para su medición. Principalmente se han centrado los estudios en población adolescente, principalmente estudiantes, derivando estos esfuerzos en la construcción de diferentes instrumentos mara medir ese uso abusivo a internet (Huang, Wang, Quin, Zhong y Tao, 2007). Se ha considerado la adolescencia como edad vulnerable, y se han señalado diversos factores presentes en esta etapa como responsables de la adicción a internet o al móvil, también se ha observado una disminución en las relaciones sociales positivas (Barros, Hernández y Vanegas, 2017), una correlación positiva entre la dependencia al móvil o internet y la impulsividad (Chambi y Sucari, 2017) y su aparición sobre todo en adolescentes que provienen de grupos sociales marginados, con escasos recurso socioeconómicos, bajos niveles de cohesión, afectividad y comunicación (Aponte, Castillo y González, 2017). Marín (2018) relaciona la adicción a internet con la disfuncionalidad familiar, apareciendo la adicción con mas frecuencia en en universitarios pertenecientes a familias con cohesión dispersa y adaptabilidad rígida y caótica. Siesquén y Aurora (2017) presentan un perfil prototípico de adolescentes que manifiestan adicción a internet, tienen entre 15-19 años, sexo masculino, proceden de la urbe y cuentan con acceso a internet tanto en el móvil como en casa. Dos de los factores de la Inteligencia Emocional analizados por Díaz y Extremera (2020) explicaban junto a la ansiedad y la adicción al móvil, los niveles de nomofobia de los sujetos.

Como efectos añadidos a la adicción a internet encontramos las conductas de ciberagresión por género. Yudes, Rey y Extremera (2019) explican dicho fenómeno unido a déficit en inteligencia emocional, y subrayan la importancia de implementar programas preventivos que consideren tanto el control parental, sobre uso de internet, como el fomento de las habilidades emocionales. También se han mostrado perfiles de inteligencia emocional diferenciados para alumnos uiversitarios y su implicación en la elección de la rama de la ciencia a estudiar (Rodrí- 
guez, Rodríguez-Góngora y Rodríguez-Rodríguez, 2020), así como su importancia en el establecimiento y desarrollo de las relaciones sociales, con una correlación positiva fuerte (Mena, 2016).

Son muchos los estudios que han abordado la importancia de implementar programas que mejoren la inteligencia emocional y de esta forma mejorar la calidad de vida de las personas. Por ejemplo, la terapia familiar sistémica aborda este tema tomando también como objeto de intervención a la adicción a internet. Plantea propuestas educativas que favorecerán una actitud activa ante los problemas adictivos y diseñan modelos comunicativos fluidos y enriquecedores (Caro y Plaza, 2016).

Teniendo en cuenta todo esto, planteamos las siguientes hipótesis de estudio:

- Las personas que puntúen más bajo en la escala de Inteligencia Emocional, obtendrán mayores niveles de abuso de las nuevas tecnologías.

- Tanto los hombres como las mujeres figurarán con niveles similares de abuso de las nuevas tecnologías, independientemente de la Inteligencia Emocional.

\section{MATERIAL Y MÉTODO}

\section{Participantes}

En el estudio han participado 547 sujetos, de los que 48 han sido eliminados por no cumplir criterios para el estudio. Del total de 499 sujetos restantes, 231 eran hombres y 268 eran mujeres, por lo que el sexo fue una variable equilibrada. La agrupación según la edad se realizó tomando intervalos con agrupación según percentiles iguales, estableciendo 5 grupos etarios (Figura 1).

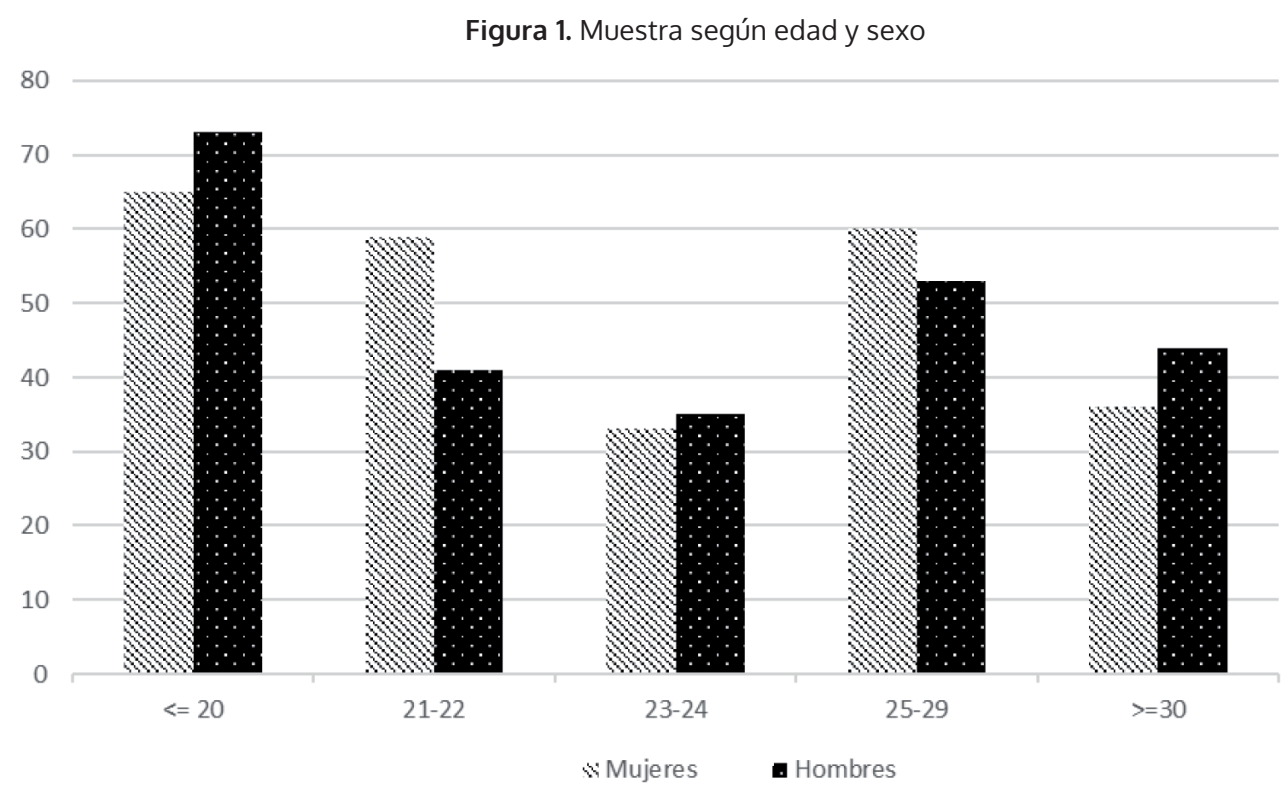




\section{Instrumentos}

En el año 2004 se inician los primeros estudios sobre el uso abusivo y, en consecuencia, generador de dependencia del móvil. Toda, Monden, Kubo y Morimoto (2004) elaboran el primer instrumento de medida para dicha posible dependencia. En España se detecta un uso elevado en jóvenes y adolescentes, y se catalogan a éstos como grupos de riesgo (Muñoz-Rivas y Agustín, 2005). Desde este inicio, y hasta 2009, encontramos varias escalas creadas con la finalidad de medir esta adicción, todas ellas con alta consistencia interna de los ítems, dando lugar a fiabilidades por encima de 0,80 .

Para la realización del estudio se han utilizado conjuntamente tres instrumentos:

- Cuestionario de Experiencias Relacionadas con Internet (CERI; Benanuy, Chamarro, Graner y Carbonell, 2009). Es una escala breve, construida con 10 ítems que puntuan sobre una escala likert de 4 puntos. Informa sobre el uso, abusivo o no, de internet. Viene determinada por dos factores, Confictos Intrapersonales, Conflictos Interpersonales.El primer factor, Conflictos Intrapersonales, incluye 6 items, que en total dan explicación al 27,14\% de la varianza, mostrando uuna consistencia interna de 0,74. El segundo factor, Conflictos Interpersonales, lo componen 4 items, que explican el $12,18 \%$ de la varianza y presentan una consistencia interna de 0,75 . El total de la escala presenta una consistencia interna de 0,77 . En nuestro estudio ha presentado una consistencia interna de 0,76.

- Cuestionario de Experiencias Relacionadas con el Móvil (CERM; Benanuy, Chamarro, Graner y Carbonell, 2009). Escala breve que consta de 10 ítems que valoran el uso, abusivo o no, del móvil. Determina dos factores: Conflictos, que incluye 5 ítems y explica el 34,6\% de la varianza, con una consistencia interna de 0,81, y Uso Comunicacional y Emociones, compuesto por otros 5 ítems que explican el 9,22\% de la varianza y aportan una consistencia interna de 0,75 . En total la escala presenta una consistencia interna de 0,80. De la misma forma, en nuestro estudio ha presentado una consistencia interna de 0,75. En conjunto las dos escalas, CERI y CERM han presentado una consistencia interna de 0,86 .

- Escala de inteligencia emocional de Wong y Law (WLEIS-S; Wong \& Law, 2002). Es una escala autoinformada que consta de 16 ítems valorados sobre una escala likert de 7 puntos. Determina cuatro factores: SEO (valoración y expresión de las emociones propias), OEA (valoración y reconocimiento de las emociones en otro, UEO (uso de las emociones) y ROE (regulación de las emociones). Cada uno de los factores está compuesto por 4 ítems. En validación al español reañlizada por Extremera, Rey y Sánchez- 
Álvarez en 2019, realizada sobre una muestra de 1460 adultos presentó un $\alpha=0,91$. En nuestra muestra ha presentado un $\alpha=0,84$.

Todos los instrumentos han sido autoinformados y se han recogido garantizando el anonimato de los sujetos.

\section{Procedimiento}

La muestra se eligió entre población universitaria. No se usaron filtors de sexo, edad o estado civil en la selección de los sujetos, pero si que se agruparon éstos con posterioridad a la recogida de datos con motivo de profundizar en esta materia mas adelante. En un primer momento se recogieron 547 conjuntos de respuestas, que tras eliminar las que contenían defectos en la cumplimentación, se quedaron en 499. A los sujetos se les informó del objeto de la presente investigación y se les garantizó el anonimato, firmando un acuerdo un acuerdo legal sujeto a la Declaración de Helsinki.

\section{Análisis de datos}

Para analizar los datos se ha procedido a clasificar a los sujetos y estimar los sujetos en conjuntos similares en cuanto al número.

Se han determinado los descriptivos para todas las variables y ha hecho comparaciones de los resultados mediante el software SPSS 25 y G-Power 3.1. Se analizaron todas las variables dependientes individualmente y en su interacción, determinando la magnitud de los efectos y la potencia de las pruebas efectuadas.

\section{RESULTADOS}

\section{Resultados sobre el uso de internet y del móvil}

Para conocer si existían diferencias significativas en los factores determinantes del uso abusivo del móvil e internet, se realizó la $t$ de Student tomando como variable independiente el sexo y como variable dependiente los diferentes factores que aportan los cuestionarios CERI y CERM. No se aprecian diferencias significativas en los resultados para ninguno de los factores analizados ni para los datos globales de ambas escalas. Sin embargo, si que aparece un efecto pequeño de la variable independiente en el factor conflictos del cuestionario CERM. 
Tabla 1. Diferencias según sexo en los factores de los cuestionarios CERI y CERM

\begin{tabular}{|c|c|c|c|c|c|c|}
\hline \multirow[b]{4}{*}{ Factor } & \multicolumn{2}{|l|}{ Sexo } & \multirow[b]{4}{*}{$t$} & \multirow[b]{4}{*}{$p$} & \multirow[b]{4}{*}{$1-\beta$} & \multirow[b]{4}{*}{ d } \\
\hline & Hombres & Mujeres & & & & \\
\hline & $\mathrm{N}=246$ & $N=253$ & & & & \\
\hline & Media (DT) & Media (DT) & & & & \\
\hline $\begin{array}{l}\text { Conflictos } \\
\text { intrapersonales }\end{array}$ & $12,23(3,13)$ & $11,96(3,31)$ & ,95 & ,71 &, 23 & ,08 \\
\hline $\begin{array}{l}\text { Conflictos } \\
\text { interpersonales }\end{array}$ & $7,33(2,06)$ & $7,04(1,89)$ & 1,65 & ,06 &, 49 & 14 \\
\hline CERI total & $19,57(4,63)$ & $19,00(4,70)$ & 1,36 & ,09 & ,38 & , 12 \\
\hline Conflictos & $7,33(2.23)$ & $6,83(2,08)$ & 2,58 & ,05 & ,82 &, 23 \\
\hline $\begin{array}{l}\text { Uso comunicacional } \\
\text { y emocional }\end{array}$ & $9,67(2,51)$ & $9,67(2,51)$ &, 03 & ,78 &, 05 & ,00 \\
\hline CERM total & $17,01(4,09)$ & $16,50(3,98)$ & 1,39 &, 93 &, 40 & , 12 \\
\hline
\end{tabular}

En la tabla 2 observamos el resultado de las comparaciones realizadas sobre los datos del CERI en función de los grupos de edad. Se observa como aparecen diferencias significativas en los resultados, tanto para conflictos intrapersonales $\left(F_{(4,494)}=2,43 ; p<0,05 ; \eta^{2}=0,13\right)$ como para conflictos interpersonales $\left(F_{(4,494)}=2,68 ; p<0,05 ; \eta^{2}=0,14\right)$ y CERI total $\left(F_{(4,494)=} 3,1 ; p<0,05 ; \eta^{2}=0,15\right)$. En las pruebas post-hoc, mediante análisis HDS de Tukey encontramos que aparecen diferencias en conflictos intrapersonales entre los grupos $<=20$ y $21-22(p=0.04)$, en conflictos interpersonales, entre los grupos $<=20$ y $21-22(p=0.02)$ y $C E R I$ total entre los grupos $<=20$ y $21-22(p=0.02)$.

Tabla 2. Diferencia de medias en los factores de CERI según grupos de edad

\begin{tabular}{llllllll}
\hline Factor & Edad & N & Media (DT) & F & p & $1-\beta$ & $\eta^{2}$ \\
\hline & $\leq 20$ & 138 & $12,78(3,06)$ & & & & \\
Conflictos & $21-22$ & 100 & $11,61(3,29)$ & & &, 13 \\
intrapersonales & $23-24$ & 68 & $11,73(3,11)$ & 2,43 &, $04^{*}$ &, 69 & \\
& $25-29$ & 113 & $12,02(3,27)$ & & & \\
& $\geq 30$ & 80 & $11,93(3,31)$ & & & \\
\hline
\end{tabular}




\begin{tabular}{|c|c|c|c|c|c|c|c|}
\hline \multirow{5}{*}{$\begin{array}{l}\text { Conflictos } \\
\text { interpersonales }\end{array}$} & $\leq 20$ & 138 & $7,59(1,83)$ & \multirow{5}{*}{2,68} & \multirow{5}{*}{, $03 *$} & \multirow{5}{*}{,73 } & \multirow{5}{*}{ 14 } \\
\hline & $21-22$ & 100 & $6,81(1,90)$ & & & & \\
\hline & $23-24$ & 68 & $6,94(2,01)$ & & & & \\
\hline & $25-29$ & 113 & $7,14(2,06)$ & & & & \\
\hline & $\geq 30$ & 80 & $7,23(2,10)$ & & & & \\
\hline \multirow{5}{*}{ CERI total } & $\leq 20$ & 138 & $20,37(4,24)$ & \multirow{5}{*}{3,11} & \multirow{5}{*}{, $01 *$} & \multirow{5}{*}{,80 } & \multirow{5}{*}{,15 } \\
\hline & $21-22$ & 100 & $18,42(4,69)$ & & & & \\
\hline & $23-24$ & 68 & $18,67(4,59)$ & & & & \\
\hline & $25-29$ & 113 & $19,15(4,80)$ & & & & \\
\hline & $\geq 30$ & 80 & $19,17(4,97)$ & & & & \\
\hline
\end{tabular}

Nota. * significativo para $p<0,05$

De la misma forma que en la escala anterior se ha procedido con la escala CERM, analizando los resultados en función de los grupos de edad. También se observa como aparecen diferencias significativas en los resultados, tanto para conflictos $\left(F_{(4,444)=} 2,68 ; p<0,05 ; \eta^{2}=0,14\right)$ como para uso comunicacional y emociones $\left(F_{(4,494)=} 2,57 ; p<0,05 ; \eta^{2}=0,14\right)$ y CERM total $\left(F_{(4,494)=} 3,47\right.$; $\left.p<0,05 ; \eta^{2}=0,16\right)$. En las pruebas post-hoc, mediante análisis HDS de Tukey encontramos que aparecen diferencias en conflictos entre los grupos $<=20$ y 21-22 ( $p=0.03)$ y CERM total entre los grupos $<=20$ y $21-22(p=0.01)$ (Tabla 3$)$.

Tabla 3. Diferencia de medias en los factores de CERM según grupos de edad

\begin{tabular}{|c|c|c|c|c|c|c|c|}
\hline Factor & Edad & $N$ & Media (DT) & $F$ & $p$ & $1-$ & 2 \\
\hline \multirow{5}{*}{ Conflictos } & $\leq 20$ & 138 & $7,50(2,05)$ & \multirow{5}{*}{2,68} & \multirow{5}{*}{, $03 *$} & \multirow{5}{*}{,74 } & \multirow{5}{*}{0,14} \\
\hline & $21-22$ & 100 & $6,69(2,19)$ & & & & \\
\hline & $23-24$ & 68 & $7,23(2,20)$ & & & & \\
\hline & $25-29$ & 113 & $6,85(2,06)$ & & & & \\
\hline & $\geq 30$ & 80 & $6,97(2,37)$ & & & & \\
\hline \multirow{5}{*}{$\begin{array}{l}\text { Uso comunicacional } \\
\text { y emociones }\end{array}$} & $\leq 20$ & 138 & $10,17(2,50)$ & \multirow{5}{*}{2,57} & \multirow{5}{*}{, $03 *$} & \multirow{5}{*}{,71 } & \multirow{5}{*}{0,14} \\
\hline & $21-22$ & 100 & $9,32(2,59)$ & & & & \\
\hline & $23-24$ & 68 & $9,83(2,54)$ & & & & \\
\hline & $25-29$ & 113 & $9,31(2,23)$ & & & & \\
\hline & $\geq 30$ & 80 & $9,62(2,66)$ & & & & \\
\hline
\end{tabular}




\begin{tabular}{|c|c|c|c|c|c|c|c|}
\hline \multirow{5}{*}{ CERI total } & $\leq 20$ & 138 & $17,67(3,72)$ & \multirow{5}{*}{3,47} & \multirow{5}{*}{, $01 *$} & \multirow{5}{*}{82} & \multirow{5}{*}{0,16} \\
\hline & $21-22$ & 100 & $16,01(4,20)$ & & & & \\
\hline & $23-24$ & 68 & $17,16(4,27)$ & & & & \\
\hline & $25-29$ & 113 & $16,17(3,62)$ & & & & \\
\hline & $\geq 30$ & 80 & $16,60(4,48)$ & & & & \\
\hline
\end{tabular}

Nota. * significativo para $p<0,05$

En el estudio del efecto que pueda ejercer la interacción de las variables independientes sobre los factores determinados por CERI y CERM, hemos encontrado que solo se observan resultados que presentan diferencias significativas en la escala que analiza el uso de internet. Aparecen resultados significativos en las dos escalas y en los datos totales, aunque la magnitud del efecto es insignificante. En las pruebas post-hoc, mediante análisis HDS de Tukey encontramos que aparecen diferencias en conflictos intrapersonales entre los grupos $<=20$ y $21-22(p=0.02)$ y CERI total entre los grupos $<=20$ y $21-22(p=0.01)$ (Tabla 4$)$.

Tabla 4. Diferencia de medias en los factores de CERM según la interacción edad*sexo

\begin{tabular}{|c|c|c|c|c|c|c|c|c|c|}
\hline \multirow[b]{2}{*}{ Factor } & \multirow[b]{2}{*}{ Edad } & \multicolumn{2}{|c|}{ Hombres } & \multicolumn{2}{|c|}{ Mujeres } & \multirow[b]{2}{*}{$F$} & \multirow[b]{2}{*}{$p$} & \multirow[b]{2}{*}{$1-\beta$} & \multirow[b]{2}{*}{$\eta^{2}$} \\
\hline & & $\mathrm{N}$ & Media (DT) & $\mathrm{N}$ & Media (DT) & & & & \\
\hline \multirow{5}{*}{ Intrapersonal } & $\leq 20$ & 73 & $12,52(2,90)$ & 65 & $13,07(3,22)$ & \multirow{5}{*}{3,33} & \multirow{5}{*}{, $01 *$} & \multirow{5}{*}{, 84} & \multirow{5}{*}{0,03} \\
\hline & $21-22$ & 41 & $12,07(2,88)$ & 59 & $11,28(3,58)$ & & & & \\
\hline & $23-24$ & 35 & $10,94(2,89)$ & 33 & $12,57(3,16)$ & & & & \\
\hline & $25-29$ & 53 & $12,49(3,35)$ & 60 & $11,60(3,16)$ & & & & \\
\hline & $\geq 30$ & 44 & $12,63(3,45)$ & 36 & $11,08(2,97)$ & & & & \\
\hline \multirow{5}{*}{ Interpersonal } & $\leq 20$ & 73 & $7,31(1,84)$ & 65 & $7,90(1,79)$ & \multirow{5}{*}{3,41} & \multirow{5}{*}{, $01 *$} & \multirow{5}{*}{, 85} & \multirow{5}{*}{0,03} \\
\hline & $21-22$ & 41 & $7,14(2,01)$ & 59 & $6,57(1,80)$ & & & & \\
\hline & $23-24$ & 35 & $6,08(2,19)$ & 33 & $7,09(1,82)$ & & & & \\
\hline & $25-29$ & 53 & $7,62(2,22)$ & 60 & $6,71(1,82)$ & & & & \\
\hline & $\geq 30$ & 44 & $7,63(2,12)$ & 36 & $6,75(1,99)$ & & & & \\
\hline \multirow{5}{*}{ CERI total } & $\leq 20$ & 73 & $19,83(4,10)$ & 65 & $20,98(4,34)$ & \multirow{5}{*}{3,97} & \multirow{5}{*}{, $00 * *$} & \multirow{5}{*}{, 91} & \multirow{5}{*}{0,03} \\
\hline & $21-22$ & 41 & $19,21(4,32)$ & 59 & $17,86(4,90)$ & & & & \\
\hline & $23-24$ & 35 & $17,74(4,64)$ & 33 & $19,66(4,38)$ & & & & \\
\hline & $25-29$ & 53 & $20,11(4,86)$ & 60 & $18,31(4,63)$ & & & & \\
\hline & $\geq 30$ & 44 & $20,27(5,24)$ & 36 & $17,83(4,33)$ & & & & \\
\hline
\end{tabular}

Nota. * significativo para $p<0,05 ;$ ** significativo para $p<0,01$ 


\section{Resultados sobre Inteligencia Emocional}

De igual forma que con las escalas CERI y CERM, se ha procedido con los resultados obtenidos mediante la escala WLEIS-S. Se han analizado todos los factores que aporta dicha escala en función de las variables independientes individualmente y en su interacción.

$\mathrm{Al}$ analizar los datos en función del sexo, se han encontrado diferencias significativas en los factores: $O E A$, que representa la valoración de las emociones de los otros, entre hombres $(M=5,38 ; D T=0,90)$ y mujeres $(M=5,68 ; D T=0,85)$, y en $R O E$, que representa la regulación de las emociones, entre hombres $(M=4,68 ; D T=1,17)$ y mujeres $(M=4,45 ; D T=1,20)$. En ambos resultados aparece un efecto pequeño según la estimación realizada con $d$ de Cohen (Tabla 5).

Tabla 5. Diferencia de medias en los factores de WLEIS-S según sexo

\begin{tabular}{|c|c|c|c|c|c|c|c|}
\hline Factor & Sexo & $\mathrm{N}$ & Media (DT) & $t$ & $p$ & 1- & $d$ \\
\hline \multirow{3}{*}{ SEA } & Hombres & 246 & $5,44(0,92)$ & \multirow{3}{*}{1,80} & \multirow{3}{*}{,07 } & \multirow{3}{*}{ 60 } & \multirow{3}{*}{0,17} \\
\hline & & & & & & & \\
\hline & Mujeres & 253 & $5,28(1,00)$ & & & & \\
\hline \multirow[b]{2}{*}{ OEA } & Hombres & 246 & $5,38(0,90)$ & \multirow[b]{2}{*}{$-3,84$} & \multirow[b]{2}{*}{, $00 *$} & \multirow[b]{2}{*}{,98 } & \multirow[b]{2}{*}{0,34} \\
\hline & Mujeres & 253 & $5,68(0,85)$ & & & & \\
\hline \multirow[b]{2}{*}{ UEO } & Hombres & 246 & $5,09(1,31)$ & \multirow[b]{2}{*}{$-0,16$} & \multirow[b]{2}{*}{, 53} & \multirow[b]{2}{*}{,07 } & \multirow[b]{2}{*}{0,02} \\
\hline & Mujeres & 253 & $5,11(1,20)$ & & & & \\
\hline \multirow[b]{2}{*}{ ROE } & Hombres & 246 & $4,68(1,17)$ & \multirow[b]{2}{*}{2,14} & \multirow[b]{2}{*}{, $03 *$} & \multirow[b]{2}{*}{,69 } & \multirow[b]{2}{*}{0,20} \\
\hline & Mujeres & 253 & $4,45(1,20)$ & & & & \\
\hline \multirow[b]{2}{*}{ IE Total } & Hombres & 246 & $5,15(0,69)$ & \multirow[b]{2}{*}{,37 } & \multirow[b]{2}{*}{,70 } & \multirow[b]{2}{*}{,95 } & \multirow[b]{2}{*}{0,02} \\
\hline & Mujeres & 253 & $5,13(0,77)$ & & & & \\
\hline
\end{tabular}

Nota. SEA= valoración de las propias emociones; OEA= Valoración de las emociones de los otros; UEO= Uso de las propias emociones; $\mathrm{ROE}=$ Regulación de las emociones, *Significativo para $p<0,05$

Se han analizado los valores de Inteligencia Emocional presentados en los diferentes factores dados por el WLEISS en los grupos de edad creados, y se ha visto la magnitud del efecto de esa variable edad sobre los mismos. En un primer análisis comparativo de las medias obtenidas se han encontrado diferencias significativas solo en el factor UEO, uso de las emociones propias, $\left(F_{(4,494)}=6,12 ; p<0,01 ; \eta^{2}=0,05\right)$ y en IE total $\left(F_{(4,494)=} 6,12 ; p<0,01 ; \eta^{2}=0,03\right)$. Aparece un efecto insignificante de la variable independiente. Al realizar las pruebas post-hoc mediante análisis HDS de Tukey encontramos que el grupo $\leq 20$ presenta diferencias significativas respecto a cada uno de los demás grupos para el factor UEO, y solo aparecen diferencias en IE total entre los grupos $\leq 20$ y $20-21$ (Tabla 6 ). 
También se ha realizado un análisis de las medias en la interacción de las variables independientes, edad y sexo, y no se han encontrado diferencias significativas en ninguno de los factores de WLEIS-S.

Tabla 6. Diferencia de medias en los factores de WLEIS-S según la edad

\begin{tabular}{|c|c|c|c|c|c|c|c|}
\hline Factor & Edad & $\mathrm{N}$ & Media (DT) & $F$ & $p$ & $1-\beta$ & $\eta^{2}$ \\
\hline \multirow{5}{*}{ SEA } & $\leq 20$ & 138 & $5,23(0,99)$ & \multirow{5}{*}{1,26} & \multirow{5}{*}{,28 } & \multirow{5}{*}{, 39} & \multirow{5}{*}{,10 } \\
\hline & $21-22$ & 100 & $5,43(1,00)$ & & & & \\
\hline & $23-24$ & 68 & $5,33(0,87)$ & & & & \\
\hline & $25-29$ & 113 & $5,36(0,98)$ & & & & \\
\hline & $\geq 30$ & 80 & $5,51(0,90)$ & & & & \\
\hline \multirow{5}{*}{ OEA } & $\leq 20$ & 138 & $5,48(0,86)$ & \multirow{5}{*}{0,56} & \multirow{5}{*}{,69 } & \multirow{5}{*}{, 18 } & \multirow{5}{*}{, 00} \\
\hline & $21-22$ & 100 & $5,54(0,83)$ & & & & \\
\hline & $23-24$ & 68 & $5,56(0,83)$ & & & & \\
\hline & $25-29$ & 113 & $5,63(0,88)$ & & & & \\
\hline & $\geq 30$ & 80 & $5,48(1,07)$ & & & & \\
\hline \multirow{5}{*}{ UEO } & $\leq 20$ & 138 & $4,68(1,29)$ & \multirow{5}{*}{6,12} & \multirow{5}{*}{, $00 * *$} & \multirow{5}{*}{,98 } & \multirow{5}{*}{, 05} \\
\hline & $21-22$ & 100 & $5,41(1,17)$ & & & & \\
\hline & $23-24$ & 68 & $5,30(1,32)$ & & & & \\
\hline & $25-29$ & 113 & $5,15(1,21)$ & & & & \\
\hline & $\geq 30$ & 80 & $5,21(1,15)$ & & & & \\
\hline \multirow{5}{*}{ ROE } & $\leq 20$ & 138 & $4,42(1,13)$ & \multirow{5}{*}{1,40} & \multirow{5}{*}{, 23} & \multirow{5}{*}{, 44} & \multirow{5}{*}{ 10 } \\
\hline & $21-22$ & 100 & $4,72(1,10)$ & & & & \\
\hline & $23-24$ & 68 & $4,48(1,19)$ & & & & \\
\hline & $25-29$ & 113 & $4,53(1,20)$ & & & & \\
\hline & $\geq 30$ & 80 & $4,73(1,36)$ & & & & \\
\hline \multirow{5}{*}{ IE Total } & $\leq 20$ & 138 & $4,95(0,72)$ & \multirow{5}{*}{3,49} & \multirow{5}{*}{, $00 * *$} & \multirow{5}{*}{,86 } & \multirow{5}{*}{,03 } \\
\hline & $21-22$ & 100 & $5,28(0,67)$ & & & & \\
\hline & $23-24$ & 68 & $5,17(0,74)$ & & & & \\
\hline & $25-29$ & 113 & $5,17(0,79)$ & & & & \\
\hline & $\geq 30$ & 80 & $5,23(0,74)$ & & & & \\
\hline
\end{tabular}




\section{Análisis de correlaciones}

En la tabla 7 observamos como los análisis de correlación de Pearson mostraron relaciones negativas entre IE total y todos los factores expresados en CERI y CERM, siendo la relación mayor con las puntuaciones totales, de forma que a menor puntuación en IE mayor puntuación en uso inadecuado del móvil o de internet. En referencia a la relación entre IE y uso de móvil, todos los factores de IE se mostraron significativos, en el uso de internet solo mostraron resultados significativos la valoración de las emociones propias y el uso y regulación de las emociones.

Tabla 7. Correlación entre variables de IE, CERI y CERM

\begin{tabular}{|c|c|c|c|c|c|c|c|c|c|c|c|}
\hline & 1 & 2 & 3 & 4 & 5 & 6 & 7 & 8 & 9 & 10 & 11 \\
\hline 1.CERI total & 1 & & & & & & & & & & \\
\hline 2.CERI Conflictos Intrapersonales &, $939^{* *}$ & 1 & & & & & & & & & \\
\hline 3.CERI Conflictos Interpersonales &, $830^{* *}$ &, $587^{* *}$ & 1 & & & & & & & & \\
\hline 4.CERM total &, $749^{* *}$ &, $667^{* *}$ &, $681^{* *}$ & 1 & & & & & & & \\
\hline 5.CERM Conflictos &, $572^{\star *}$ &, $441^{* *}$ &, $630^{* *}$ & $840^{* *}$ & 1 & & & & & & \\
\hline $\begin{array}{l}\text { 6.CERM Uso comunicacional } \\
\text { y emociones }\end{array}$ &, $712^{* *}$ &, $693^{* *}$ &, $552^{* *}$ & $883^{* *}$ & $488^{* *}$ & 1 & & & & & \\
\hline 7.IE_total &,$- 232^{* *}$ &,$- 205^{* *}$ &,$- 214^{* *}$ &,$- 343^{* *}$ &,$- 262^{* *}$ &,$- 325^{* *}$ & 1 & & & & \\
\hline $\begin{array}{l}\text { 8.Valoracion de las propias } \\
\text { emociones }\end{array}$ &,$- 162^{* *}$ &,$- 129^{* *}$ &,$- 171^{\star *}$ &,$- 290^{* *}$ &,$- 259^{* *}$ &,$- 242^{* *}$ &, $727^{\star \star}$ & 1 & & & \\
\hline $\begin{array}{l}\text { 9.Valoracion de las emociones } \\
\text { de los otros }\end{array}$ &,- 055 &,- 032 &,- 078 &,$- 102^{*}$ &,$- 128^{* *}$ &,- 054 &, $511^{* *}$ &, $254^{* *}$ & 1 & & \\
\hline 10.Uso de las propias emociones &,$- 194^{* *}$ &,$- 188^{* *}$ &,$- 151^{* *}$ &,$- 216^{* *}$ &,$- 154^{* *}$ &,$- 214^{* *}$ &, $746^{* *}$ &, $369^{* *}$ &, $193^{\star *}$ & 1 & \\
\hline 11.Regulacion de emociones &,$- 199^{* *}$ &,$- 181^{* *}$ &,$- 176^{* *}$ &,$- 311^{\star *}$ &,$- 182^{* *}$ &,$- 344^{* *}$ &, $723^{* *}$ &, $414^{* *}$ &, $115^{*}$ &, $352^{* *}$ & 1 \\
\hline$x$ & 19,28 & 12,09 & 7,18 & 16,76 & 7,08 & 9,67 & 5,14 & 5,36 & 5,54 & 5,10 & 4,57 \\
\hline DT & 4,67 & 3,22 & 1,98 & 4,04 & 2,17 & 2,51 & ,74 &, 96 &, 88 & 1,26 & 1,19 \\
\hline
\end{tabular}

Nota. ** $p<0,01 ; * p<0,05 ; X=$ Media; DT= Desviación Típica

Para profundizar en el estudio, se realizó un análisis de la influencia de la Inteligencia Emocional, como covariable, sobre el uso abusivo de internet y del móvil, teniendo en cuenta las variables independientes sexo y edad. Se observó una relación significativa en cuanto al uso abusivo del móvil y la IE en relación al sexo $\left(F_{(1,496)}=5,43 ; p=, 02 ; \eta^{2}=, 01 ; 1-\beta=, 63\right)$, presentando un efecto insignificante de la variable independiente (Tabla 8). 
Tabla 8. Efecto de la IE sobre el uso abusivo del móvil e internet

\begin{tabular}{lllllllllll}
\hline \multicolumn{10}{c}{ CERI } & \multicolumn{10}{c}{ CERM } \\
\hline Covariable & VI & $F$ & $p$ & $\eta^{2}$ & $1-\beta$ & $F$ & $p$ & $\eta^{2}$ & $1-\beta$ \\
\hline \multirow{2}{*}{ IE } & Sexo & 3,15 &, 07 &, 00 &, 42 & 5,43 &, $02^{*}$ &, 01 &, 63 \\
& Edad & 40,7 &, 09 &, 01 &, 60 & 2,02 &, 09 &, 01 &, 60 \\
\hline
\end{tabular}

Nota. * significativo para $\mathrm{p}<0,05$

\section{DISCUSIÓN}

En los diferentes estudios revisados sobre la adicción a internet o al móvil se presentan resultados en los que aparecen en mayor o menor grado índices de dependencia, con disminución de las relaciones sociales (Barros et al., 2017; Mena, 2016), presencia de conductas impulsivas (Chambi y Sucari, 2017) y asociación con conductas como la ciberagresión (Yules, Rey y Extremera, 2019). En nuestro estudio todos los sujetos han mostrado niveles normalizados sobre el abuso de internet y el móvil, por lo que no hemos podido realizar una relación con estas conductas, aunque si se hayan visto diferenciadas en función de categorías como el sexo o la edad. Los niveles de inteligencia emocional presentados son medios-altos en gran parte de los sujetos. No hay suficiente muestra con Inteligencia Emocional baja como para analizar en profundidad, y con claridad, la influencia que tendría con las conductas disruptivas ya mencionadas.

El principal objetivo de esta investigación era comprobar la existencia de una relación entre la IE y el uso abusivo de internet y/o el móvil. En los resultados obtenidos se ha podido observar como los valores ofrecidos sobre el uso abusivo de internet y del móvil están por debajo de los presentados en el estudio de creación de los cuestionarios CERI y CERM (Fagues, M., Chamarro, A., Jordania, C. y Carbonell, X. (2009). Hemos comprobado como ninguno de los grupos creados por los niveles de las variables independientes han mostrado valores anormales respecto al uso abusivo de internet y/o móvil, presentando todos valores compatibles con un uso normal y apropiado.

En referencias a los valores obtenidos en IE, comparados con los obtenidos por Extremera, Rey y Sanchez-Álvarez (2019) en su validación en población española del WLEIS-S, si que observamos valores de IE más altos tanto para hombres como para mujeres en los factores SEA y OEA, que hacen referencia a la valoración de las emociones propias y de los otros. Sin embargo, en cuanto al uso de las emociones (UEO) observamos valores altos en hombres y bajos en mujeres. En regulación de emociones se objservan valores bajos en ambos sexos. Ya en el análisis del valor global de IE, hemos observado valores medios superiores a la media, tanto en hombres como en mujeres. 
En el análisis correlacional se ha observado como la IE estaba relacionada con el uso internet y el móvil de forma negativa, a pesar de haberse presentado valores bajos en adicción y altos en IE. No obstante, solo se ha visto un efecto significativo en cuanto al uno del móvil en función del sexo, donde los hombres han mostrado valores mas altos en CERM total y en IE total que las mujeres.

A la luz de esta investigación se hace necesaria una profundización en este estudio, para lo que se propone:

1. El uso de cuestionarios mas amplios y que evalúen un abanico mayor de áreas, que puedan ratificar o aclarar más en profundidad los resultados obtenidos.

2. Replicar este estudio en otro ambito social, con el fin de comprobar si existen resultados compatibles.

3. El apego y la Inteligencia emocional han mostrado estar relacionados (Rodríguez, 2018). Se propone una linea de investigación que una estas tres variables, para estudiar una posible correlación entre ellas.

\section{CONCLUSIONES}

Teniendo en cuenta el punto de partida y los resultados obtenidos, concluimos que:

- A medida que aumenta el uso abusivo de las nuevas tecnologías, los niveles de Inteligencia Emocional, que aparecen en los sujetos, son menores. Debiendo determinar los agentes causa y efecto.

- Apenas existen diferencias en Inteligencia Emocional entre hombres y mujeres, a excepción de en la valoración de las emociones de los otros donde las mujeres puntúan más alto y en regulación de las emociones, donde los hombres puntúan más alto. Se podría decir que las mujeres atienden mejor a las emociones de los demás y los hombres hacen una mejor regulación de las emociones en general.

\section{REFERENCIAS BIBLIOGRÁFICAS}

Aponte Rueda, Deyvar R., Castillo Chávez, Paola, \& González Estrella, José E. (2017). Prevalencia de adicción a internet y su relación con disfunción familiar en adolescentes. Revista Clínica de Medicina de Familia, 10(3), 179-186. http://scielo.isciii.es/scielo.php?script=sci arttext\&pid=S1699$\underline{695 X 2017000300179 \& \operatorname{lng}=\mathrm{es} \& \mathrm{t} \operatorname{lng}=\mathrm{es}}$. 
Barros, S., Hernández, Y., Vanegas, O.S., Cedillo, L., Alvarado, H. y Cabrera, J.A. (2017). Adolescentes de básica superior con adicción a internet y redes sociales y relaciones interpersonales. Revista Electrónica de Psicología Iztacala, 20(3), 42-68. http://revistas.unam.mx/index.php/repi/article/ view/61775

Caro, C. y Plaza, J. (2016). Intervención educativa familiar y terapia sistémica en la adicción adolescente a Internet-Intervención educativa familiar y terapia sistémica en adicción a internet adolescente. REOP - Revista Española de Orientación y Psicopedagogía, 27(1), 99-113. https://doi. org/10.5944/reop.vol.27.num.1.2016.17031

Chambi, S., y Sucari, B. A. (2017). Adicción a internet, dependencia al móvil, impulsividad y habilidades sociales en pre-universitarios de la Institución Educativa Privada Claudio Galeno, Juliaca 2017. Juliaca: Imprenta Unión. http://repositorio.upeu.edu.pe/handle/UPEU/664

Diaz, N. y Exytremera, N. (2020). Inteligencia emocional, adicción al smartphone y malestar psicológico como predictores de la nomofobia en adolescentes. Know and Share Psychology, 1(2), 7-13. http://dx.doi.org/10.25115/kasp.v1i2.3195

Fargues, M., Chamarro, A., Jordania, C. y Carbonell, X. (2009). Validation of two brief scales for Internet addiction and mobile phone problem use. Psicothema. 21. 480-485. http://www.psicothema. com/pdf/3658.pdf

Huang Z, Wang M, Qian M, Zhong J, Tao R. (2007). Chinese Internet addiction inventory: developing a measure of problematic Internet use for Chinese college students. Cyberpsychol Behav.,10(6), 805-811. https://doi.org/10.1089/cpb.2007.9950

Lluén, R.I. (2017). Características de la adicción a internet en estudiantes de nivel secundario en colegios estatales en el distrito de Chiclayo. Tesis de pregrado. Universidad de San Martín de Porres. Perú. http://repositorio.usmp.edu.pe/handle/usmp/2539

Marin, C. (2018). Adicción a internet y funcionalidad familiar en universitarios de Lima Norte. CASUS: Revista de Investigación y Casos en Salud, 3(1), 1-8. https://dialnet.unirioja.es/servlet/ articulo?codigo $=6538963$

Mena-Olmedo, R. (2016). Inteligencia emocional y relaciones sociales. TFG. Universidad de Jaén. España. https://hdl.handle.net/10953.1/3540

Rodríguez, J. C. (2018). Apego e Inteligencia emocional en familias en riesgo social. Editorial Académica Española.

Rodríguez Rodríguez, J. C., Rodríguez Góngora, J., \& Rodríguez Rodríguez, J. A. (2020a). Inteligencia emocional en universitarios: análisis descriptivo en alumnos de $1^{\circ} \mathrm{y} 2^{\circ}$ de Grado. Know and Share Psychology, 1(1), 23-37. https://doi.org/10.25115/kasp.v1i1.3081 
Yudes, C., Rey, L. y Extremera, N. (2019). Ciberagresión, adicción a internet e inteligencia emocional en adolescentes: un análisis de diferencias de género. Voces de la Educación, 27-44. https://hal. archives-ouvertes.fr/hal-02511693 